\title{
Complémentarité et équilibre de l'apport alimentaire en protéines et en lipides
}

\section{Nature and level of dietary proteins and lipids in food intake}

Oléagineux, Corps Gras, Lipides. Volume 10, Numéro 1, 31-40, Janvier - Février 2003, Protéines et lipides végétaux : interactions nutritionnelles et fonctionnelles

Auteur(s) : Jacques BELLEVILLE, UPRES Lipides et Nutrition, Université de Bourgogne Faculté des Sciences Gabriel, 6, bd Gabriel 21000 Dijon, France.

\section{Author(s) : Jacques BELLEVILLE}

Summary : The nature and level of dietary protein is liable to influence cholesterol and essential fatty acid (EFA) metabolism. Dietary vegetable protein, particularly the undigested fraction, decreases intestinal cholesterol absorption, increases the faecal excretion of steroids, and enhances the catabolism of cholesterol-carrying lipoproteins by increasing the activity or number of LDL receptors. However, the hypocholesterolemic effect of protein may be due to the presence of non-protein components and hence the purity degree of the selected protein, and the concomitant addition or not of cholesterol. Dietary proteins with different amino acid composition may modulate the secretion of hormones (e.g. glucagon and insulin), which in turn may modify the activity of enzymes responsible for cholesterol metabolism (e.g. HMG-CoA reductase, 7alpha-hydroxylase, lecithin: cholesterol acyl transferase) and polyunsaturated fatty acid synthesis (e.g. Delta5 and Delta6desaturases). PUFA affect membrane fluidity and the synthesis of eicosanoids: prostaglandins and leukotrienes. Protein deficiency affects severely the PUFA amounts, particularly arachidonic and EPA acids which are precursors of prostaglandins, thromboxanes and leukotrienes, and the possible consequences of their diminution may be an underlying cause of some of the common clinical symptoms in protein malnutrition, such as hair loss, dry scaly dermatitis and increased water permeability. Protein deficiency may thus increase the EFA requirement and precipitate marginal EFA deficiency. The dietary protein level may also influence the efficiency of antioxidant system and/or the release of reactive oxygen species. Tissue lipid peroxidation could be markedly enhanced by feeding low-protein diets, probably due to a depressed efficiency of antioxidative enzyme activities.

Keywords : soy protein, casein, cholesterol, essential fatty acid, peroxidation, protein malnutrition.

\section{ARTICLE}

Brefs rappels sur le métabolisme des acides gras essentiels

Les acides gras essentiels (AGE) constituent un groupe d'acides gras qui ne peut être synthétisé de novo par I'homme et les animaux. Il existe deux AGE fondamentaux, l'acide linoléique (18:2n-6) et l'acide alpha-linolénique (alpha-18:3n-3) à partir desquels des acides gras ayant 20 ou 22 atomes de carbone et contenant jusqu'à 6 doubles liaisons sont métaboliquement dérivés. Les acides gras 
polyinsaturés (AGPI), dérivés des AGE par désaturation et élongation, apparaissent dans les tissus comme des composés essentiels, et en conséquence une modification de leur métabolisme peut perturber la structure et la fonction des membranes cellulaires et entraîner des altérations permanentes. Comme le montre la figure 1 la synthèse des AGPI à partir des deux AGE fondamentaux utilise les mêmes enzymes et en particulier les désaturases. Le $18: 3 n-3$ s'est révélé être un substrat privilégié par rapport au $18: 2 n-6$ pour la Delta6-désaturase ce qui participe à la compétition entre les deux familles n-3 et n-6, et un excès alimentaire de l'un des deux AGE peut perturber la synthèse des AGPI dérivés de l'autre [1]. Le tableau I indique que certainsAGPI sont la source de différentes familles d'éicosanoïdes : leucotriènes et prostaglandines qui jouent des rôles physiologiques nombreux et importants. Des études réalisées chez des animaux et des sujets humains ont montré de nombreuses relations entre un apport alimentaire adéquat en protéines et le métabolisme des AGE $[2,3]$.

Effet hypocholestérolémiant des protéines végétales

Lorsque les études portent sur des protéines végétales, en général ce sont les protéines de soja (PS) qui sont choisies comme modèle, et la caséine représente en général le modèle des protéines animales, c'est pourquoi les comparaisons sont souvent établies entre ces deux types de protéines.

Les protéines alimentaires sont d'importants régulateurs du niveau de cholestérol plasmatique. Les PS, comparées à la caséine, exercent un effet hypocholestérolémiant significatif chez diverses espèces animales [4-15] et chez l'homme [11, 16-20], mais chez l'homme, cet effet n'est présent que lorsque le régime apporte suffisamment de cholestérol ou que les patients sont hypercholestérolémiques. Ainsi, des enfants hypercholestérolémiques qui reçoivent des PS voient leur taux de cholestérol plasmatique diminuer nettement en 8 semaines [21]. Le mécanisme de cet effet est encore l'objet de controverse. La caséine, au contraire, a un effet hypercholestérolémiant en augmentant l'absorption du cholestérol et en diminuant son temps de renouvellement [22]. Plusieurs études suggèrent que l'effet hypocholestérolémiant des protéines végétales, particulièrement des PS, serait dû en grande partie a une plus forte excrétion fécale des stérols, en raison d'une réduction de leur absorption intestinale [23]. C'est un effet qui ressemble à celui obtenu avec certaines fibres alimentaires. Afin de compenser les pertes fécales des stérols, les PS peuvent stimuler les activités hépatiques de l'hydroxy méthylglutaryl CoA (HMG-CoA) réductase [23], enzyme limitante dans la biosynthèse du cholestérol, et de la cholestérol 7alpha-hydroxylase, enzyme clef qui permet la transformation du cholestérol en acides biliaires [24, 25]. Iwani et al. [26] indiquent que des peptides hydrophobes, présents dans les PS, et qui persistent après leur digestion, se lient aux acides biliaires et réduisent leur absorption, ce qui accroît la perte fécale du cholestérol. L'absorption du cholestérol et des acides biliaires dépendrait donc des propriétés physicochimiques des protéines ou de leurs produits de digestion [7, 27-29]. Il a été ainsi montré que la partie non-digeste des PS est plus hypocholestérolémiante que les PS totales avec des régimes enrichis en cholestérol [27-29] ou des régimes sans cholestérol $[10,29]$. Cependant, si une plus forte perte fécale de stérols peut augmenter la production d'acides biliaires et diminuer la concentration de cholestérol sérique, cela n'entraîne pas automatiquement cette baisse de concentration [30]. En effet, Madani et al. [31] montrent que le taux de cholestérol plasmatique chez le rat n'est pas modifié par l'origine des 
protéines (PS hautement purifiées ou caséine), si le régime est dépourvu de cholestérol. Dans une autre étude [32], les mêmes auteurs indiquent qu'avec $(0,1 \%)$ ou sans cholestérol dans le régime, en utilisant les mêmes protéines, il n'y a pas de modification du cholestérol et des triglycérides (TG) plasmatiques, bien que les pertes fécales des stérols neutres et acides soient augmentées avec les PS hautement purifiées. Cela peut être dû au fait que la forte purification des PS minimise l'augmentation de l'excrétion fécale des stérols [32]. L'apport de cholestérol dans les régimes diminue l'activité de la HMG-CoA réductase, et davantage avec les PS par rapport à la caséine [32]. En l'absence de cholestérol alimentaire les PS hautement purifiées diminuent l'activité de la 7alphahydroxylase, et augmentent cette activité en présence de $0,1 \%$ de cholestérol [32], alors que Choi et al. [33] ne montraient pas de différence au niveau de cette activité avec les PS ou la caséine, avec ou sans cholestérol dans les régimes. Les régimes supplémentés avec $0,1 \%$ de cholestérol augmentent les concentrations de cholestérol et de TG dans le foie des rats avec les deux types de protéines, la caséine entraînant des valeurs plus élevées. Lee et al. [34] obtiennent des résultats semblables avec des apports en cholestérol variant de 0,05 à $1 \mathrm{~g} / 100 \mathrm{~g}$ de régime, le cholestérol sérique est peu modifié, tandis que le cholestérol hépatique l'est d'une façon dose dépendante. Les isolats de petit lait ont aussi un effet hypocholestérolémiant par rapport à la caséine, cet effet est plus puissant que celui des PS $[35,36]$.

II a été aussi suggéré que l'effet hypocholestérolémiant des PS pouvait être dû à leurs teneurs en composants non protéiques, donc pouvait varier selon le degré de pureté des PS. Des composés nonprotéiques (fibres, acide phytique, minéraux, isoflavones ...), associés aux PS, peuvent en effet aussi modifier le métabolisme du cholestérol et avoir un effet hypocholestérolémiant [23, 37-39], c'est le cas en particulier des isoflavones, mais celles-ci ne participeraient qu'en partie à l'effet hypocholestérolémiant des PS [40]. Cependant, Anthony et al. [41] montrent que les isoflavones de PS, mais pas les PS débarrassées de leurs isoflavones, diminuent le cholestérol plasmatique chez le singe rhésus.

Rôle de la composition en acides aminés des protéines végétales

Saeki et Kiriyama [42] indiquent que c'est plutôt la différence de composition en acides aminés des PS et de la caséine qui serait le principal facteur modifiant la teneur du cholestérol plasmatique. Cependant, un mélange d'acides aminés copiant la composition des PS est moins hypocholestérolémiant que la protéine elle-même, et aucune différence significative n'est constatée dans l'absorption et l'excrétion du cholestérol chez des rats recevant le mélange d'acides aminés correspondant aux PS ou à la caséine [23]. Cela peut être dû au fait qu'il existe une différence dans la biodisponibilité des acides aminés libres comparée à celle de la protéine ce qui peut expliquer que leurs actions ne soient pas semblables [23, 38, 43].

Les PS, comparées à la caséine, sont plus riches en glycine, alanine, arginine et cystéine et plus pauvres en méthionine [44]. Les PS contiennent moitié moins de méthionine et la méthionine augmente le cholestérol sérique [45]. Lorsque l'on supplémente les PS avec de la méthionine, le cholestérol plasmatique augmente [46] et il existe une corrélation positive entre la teneur en 
méthionine de la protéine et le taux de cholestérol circulant. Cependant, l'ajout de méthionine aux PS n'empêche pas la persistance d'un effet hypocholestérolémiant; seulement $40 \%$ de l'effet hypocholestérolémiant des PS serait dû à leur faible teneur en méthionine [46]. Les teneurs en glycine sont de $3,6 \mathrm{~g} / 100 \mathrm{~g}$ dans les PS et de $1,9 \mathrm{~g} / 100 \mathrm{~g}$ dans la caséine et le rapport méthionine/glycine est de 2,3 pour la caséine et de 1,2 pour les PS ; quand on ajoute de la glycine à la caséine chez le rat le cholestérol sérique diminue [45]. Chez le cochon d'Inde, le fait de supplémenter la caséine en glycine, alanine, arginine et cystine [15] réduit le cholestérol plasmatique et en particulier le LDL-cholestérol. Ceci rejoint les résultats de plusieurs études qui montrent une corrélation négative chez le rat entre le niveau de cholestérol sérique et l'apport en alanine [47], en arginine [48] et en cystine [47, 49]. Le rapport lysine/arginine est de 2 pour la caséine et de 0,8 pour les PS. Morita et al. [50] mettent en évidence une relation positive entre le cholestérol sérique et le rapport lysine/arginine. Chez le rat recevant un régime sans cholestérol, il existe une corrélation positive entre le cholestérol sérique et l'apport alimentaire en acide glutamique, méthionine, proline et tyrosine $[47,48]$.

Par ailleurs, les PS diminuent le rapport $\mathrm{CD}^{+} / \mathrm{CD}^{+}$dans les cellules $\mathrm{T}$ de la rate ce qui peut diminuer la production des IgA, IgE, IgG [23], au contraire des protéines animales (caséine, isolats de petit lait) [35]. Une partie de ces actions peut être attribuée à la modulation d'hormones telles que le glucagon qui varie en fonction du rapport arginine/lysine des protéines qui est de 0,48 pour la caséine et de 1,07 pour les PS [51, 52].

Protéines végétales et enzymes du métabolisme du cholestérol

Les PS augmentent l'apport en arginine et l'augmentation du taux d'arginine plasmatique accroît la libération de glucagon [52] et le glucagon inhibe l'activité de la HMG-CoA réductase [53]. II a été montré que l'activité de la HMG-CoA réductase est plus basse chez les rats recevant un mélange d'acides aminés simulant les PS qu'avec un mélange simulant la caséine [23]. La différence entre une protéine intacte et le mélange d'acides aminés est attribuée à la présence d'une fraction indigeste dans les protéines végétales. L'Acyl Coenzyme A : Cholestérol Acyltransférase (ACAT) est une enzyme microsomale qui permet la synthèse d'esters de cholestérol dans la cellule. L'ACAT joue un rôle important dans le transport du cholestérol de l'intestin aux lipoprotéines et dans la régulation du métabolisme du cholestérol dans le foie. L'activité de l'ACAT est réduite significativement avec les PS comparées à la caséine [54].

La Lécithine: Cholestérol Acyltransférase (LCAT) est une enzyme située à la surface des HDL. C'est l'enzyme la plus importante dans le plasma qui transforme le cholestérol libre des HDL en esters de cholestérol qui migrent au cœur des HDL, les HDL facilitant le transport du cholestérol des tissus extrahépatiques vers le foie. Forsythe et al. [13] ont montré, chez le porc, que le régime caséine réduit l'activité de la LCAT ce qui peut réduire l'élimination du cholestérol et entraîner son accumulation dans les tissus périphériques. Cependant, Park et Liepa [14] mettent en évidence une augmentation de l'activité de la LCAT sérique chez des rats recevant des protéines animales comparés à ceux recevant des protéines végétales. Sugano et al. [55] ont mis en évidence que chez 
des rats recevant des PS les concentrations d'apo A1 étaient diminuées, et comme l'apo A1 est un activateur de la LCAT, cela peut expliquer sa plus faible activité.

Protéines végétales et métabolisme des acides gras polyinsaturés

Les effets des AGPI sur le métabolisme lipidique sont connus, mais les actions dépendent du type d'AGPI, par exemple l'acide linoléique réduit le niveau du cholestérol sanguin [56], tandis que les AGPI en n-3 diminuent l'agrégation plaquettaire [57] et les triglycérides plasmatiques [58]. D'une façon générale les AGPI et les PS diminuent le taux de cholestérol plasmatique par diminution de son absorption intestinale et de sa synthèse et par augmentation de son utilisation, de sa dégradation et de son excrétion [51]. L'acide alpha-linolénique (18:3n-3) peut aussi inhiber compétitivement la conversion de l'acide linoléique en acide arachidonique, puisque ces acides gras sont en compétition vis à vis des mêmes systèmes enzymatiques pour leur élongation et leur désaturation [59]. Ainsi les AGPI en $n-3$ suppriment l'inflammation en réduisant la synthèse du $20: 4 n-6$, fournisseur d'éicosanoïdes proinflammatoires, à partir des phospholipides membranaires [60-62]. L'huile de périlla (riche en $18: 3 n-3$ ) possède ainsi un rôle antiinflammatoire [63]. Le $18: 2 n-6$ et le $18: 3 n-$ 3 inhibent la désaturation et l'élongation des autres familles d'AGPI et l'inhibition compétitive est plus forte avec le $18: 3 n-3$ qu'avec le $18: 2 n-6$ [64]. L'huile de perilla diminue les lipides sériques par rapport à l'huile de carthame (riche en $18: 2 n-6$ ) et l'huile de bourrache (riche en $18: 3 n-6)$. Chez le rat le $18: 3 n-3$ supprime l'expression du gène de l'enzyme lipogénique [65] et stimule l'oxydation des acides gras dans le foie [60].

Non seulement les lipides alimentaires, mais aussi le type et la quantité de protéines consommées peuvent agir sur le métabolisme des lipides [31,66]. C'est ainsi qu'une combinaison adéquate de lipides associée aux PS peut entraîner un effet favorable sur le métabolisme lipidique et la production d'éicosanoïdes [63]. Sakono et al. [6] combinent différentes protéines avec des huiles variées, ils montrent que l'huile de perilla riche en alpha-linolénique, diminue le cholestérol sérique par rapport à l'huile de carthame riche en $18: 2 n-6$ et que c'est l'association PS + huile de périlla qui se révèle la plus hypocholestérolémiante [6]. Donc, l'huile et la protéine exercent un effet synergique. L'effet hypocholestérolémiant de l'huile de perilla ne s'accompagne pourtant pas d'une augmentation de la perte fécale de stérols. Le $18: 3 n-3$ entraîne aussi une baisse des TG plasmatiques, les protéines n'ayant pas d'effet, par contre la baisse constatée des TG hépatiques est essentiellement due aux PS. Les niveaux de TG hépatiques sont plus faibles chez les rats recevant des isolats de PS que chez ceux recevant de la caséine ou des isolats de petit lait [6].

Les protéines alimentaires modifient le métabolisme des AGE [51]. Chez le rat, les PS, comparées à la caséine, réduisent dans les microsomes hépatiques, l'activité de la Delta6n-6-désaturase [67]. Le plus faible rapport $20: 4 n-6 / 18: 2 n-6$, index de désaturation du linoléate, qui est observé dans les microsomes hépatiques de rats reçevant des PS, comparés à ceux reçevant de la caséine, a été attribué à une réduction de l'activité de cette enzyme [27, 29, 33]. L'effet des PS sur l'activité de la Delta6-désaturase peut s'exercer par l'intermédiaire du glucagon qui est augmenté par l'arginine, apportée en quantité importante par les PS, et le glucagon inhibe et l'insuline stimule l'activité de la Delta6-désaturase. Cependant, les récepteurs à l'insuline seraient stimulés par les PS en présence d'acides gras saturés et les PS peuvent diminuer la résistance à l'insuline, mais seulement quand les 
rats reçoivent peu d'AGPI [68]. D'une façon générale, toutes les hormones ayant une activité hyperglycémiante diminuent l'activité des désaturases (noradrénaline, glucagon, corticoïdes, thyroxine) [69].

Les PS ont aussi la capacité d'accroître la fluidité des membranes microsomiales hépatiques, alors que la diminution de leur fluidité augmente l'activité de la Delta6-désaturase qui accroît les teneurs membranaires en AGPI et ainsi rétablit la fluidité normale.

L'activité des désaturases qui catalysent la biosynthèse des AGPI, tels que l'acide arachidonique (AA) et l'acide docosahexaénoïque (DHA), est aussi influencée par la quantité et la qualité des protéines alimentaires [70-72]. C'est pourquoi les protéines alimentaires influencent les niveaux de AA, DHA, et des autres AGPI à longues chaînes en n-6 et n-3 dans les tissus de rats. Les régimes hyperprotéiques, quand la teneur en protéines est supérieure à $35 \%$, augmentent l'activité de la Delta6-désaturase [73]. Un régime riche en protéines apportant $45 \%$ de l'énergie sous forme de caséine augmente l'activité de la Delta6-désaturase dans les microsomes de foie de rat comparé à un régime apportant seulement $5 \%$ de l'énergie sous forme de protéines [72], ce qui suggère que des enfants déficients en protéines peuvent développer une carence en AGPI comme nous le verrons plus loin [74].

La conversion de l'acide linoléique (18:2n-6) en acide arachidonique $(20: 4 n-6)$ est diminuée chez des rats consommant des protéines végétales, en particulier des PS, en comparaison avec la caséine [6]. Cet effet est dû à une diminution de l'activité de la Delta6-désaturase des microsomes de foie chez les rats recevant des PS, comparés à ceux recevant de la caséine [10,67]. De nombreux auteurs suggèrent que le métabolisme de l'acide linoléique est principalement régulé par le contenu en arginine de la protéine [51, 75-77]. L'addition d'arginine à la caséine augmente les teneurs en acide linoléique et diminue celles en acide arachidonique, alors que l'ajout de lysine ne modifie rien [76, 77].

En agissant sur le métabolisme des acides gras, en diminuant l'activité de la Delta6-désaturase, les PS modifient la production d'éicosanoïdes, par exemple elles diminuent les $\mathrm{PGI}_{2}$ de l'aorte [78].

La présence ou non de cholestérol dans le régime doit aussi être prise en compte, car le cholestérol inhibe aussi la transformation de l'acide linoléique en acide arachidonique $[51,79]$. Lorsque le régime est dépourvu de cholestérol les PS diminuent les activités des Delta6n-6 et Delta6n-3-désaturases, alors que la Delta5n-6-désaturation n'est pas modifiée par la source de protéines [32]. Ces modulations se font par modification de la fluidité membranaire, les PS en réduisant la fluidité membranaire diminuent l'activité de la Delta6-désaturase [67]. Lorsque le régime contient du cholestérol c'est la caséine qui diminue le plus l'activité de la Delta6-désaturase [32].

Donc les PS hautement purifiées n'ont pas d'effet sur le niveau du cholestérol plasmatique, mais augmentent l'activité de la HMG-CoA réductase et l'excrétion fécale des stérols, et diminuent la conversion du $18: 2 n-6$ en $20: 4 n-6$ [32]. Il a même été suggéré qu'une protéine qui augmente le cholestérol sérique accroît la biosynthèse des AGPI 20 : 4n-6 et 22-6n-3. 
Les PS augmentent, dans les phospholipides hépatiques, les teneurs en acide linoléique et le $20: 3 n$ 6 et diminuent celle du $20: 4 n-6$ et les rapports $20: 4 n-6 / 18: 2 n-6,20: 4 n-6 / 20: 3 n-6$ et $20: 3 n-$ $6+20: 4 n-6 / 18: 2 n-6$ (indices de désaturation de l'acide linoléique) dans les phospholipides de foie, et cela est surtout net chez les jeunes rats $[35,80]$, ce qui ressemble aux résultats obtenus chez des animaux carencés en protéines [81]. Cela provient peut être du fait que les protéines végétales sont relativement pauvres en certains acides aminés essentiels.

Noguchi et al. [82] et Ikeda et al. [83] indiquent que le métabolisme de l'acide alpha-linolénique (18:3n-3) est aussi modifié différemment suivant le type de protéine consommé. En particulier la transformation de l'acide alpha-linolénique en EPA $(20: 5 n-3)$ puis en DHA $(22: 6 n-3)$ dans les phospholipides microsomiaux de foie est significativement diminuée chez les animaux consommant des PS comparés à la caséine [82]. Cependant, ils montrent que la conversion métabolique de l'EPA en DPA (22: $5 n-3)$ est augmentée chez les rats recevant les PS, mais qu'inversement la conversion du DPA en DHA est diminuée.

Les PS, comparées à la caséine, contiennent moins de méthionine, Terasawa et al. [84] supplémentent on non des PS avec de la méthionine et montrent que la proportion de $20: 5 n-3$ dans les fractions phosphatidyléthanolamines et phosphatidylcholines hépatiques est diminuée dans le groupe de rats PS comparé à celui recevant de la caséine, avec ou sans supplément en méthionine. La proportion de $22: 5 n-3$ est significativement plus faible dans les mêmes phospholipides avec les PS non supplémentées en méthionine, mais elle est augmentée significativement avec le supplément. Les valeurs des deux rapports $22: 5 n-3+22: 6 n-3 / 20: 5 n-3$ et $22: 5 n-3 / 20: 5 n-3$ sont significativement augmentées avec les PS supplémentées ou non avec de la méthionine, et le rapport $20: 3 n-6+20: 4 n-6 / 18: 2 n-6$ diminue significativement avec les PS, avec ou sans supplément en méthionine, et les proportions de $22: 5 n-3$ et de $18-2 n-6$ sont plus élevées dans les groupes PS que dans les groupes caséine. Ces résultats suggèrent que le processus de transformation du $20: 5 n-3$ en 22 : 6n-3 est accéléré dans le groupe PS supplémenté en méthionine et que l'élongation du $20: 5 n-3$ en 22 : 5n-3 est facilitée. Cependant, les rats recevant des PS ont un poids inférieur à ceux recevant de la caséine en l'absence de supplément en méthionine et il est possible que l'utilisation du $20: 5 n$ 3 à des fins énergétiques soit alors majorée. Il est à noter que la relation n'est pas étroite entre les teneurs en AGPI et les activités des désaturases. En effet, Ulmann et al. [85] ont montré qu'il n'y a pas de corrélation entre les activités des désaturases et la composition en acides gras des phospholipides microsomiaux, car d'autres facteurs entre en jeu, l'élongation, l'oxydation, la disponibilité du substrat et l'utilisation des métabolites formées.

Protéines et métabolisme des lipoprotéines

Les PS peuvent aussi diminuer le cholestérol plasmatique en réduisant, au niveau du foie, la formation de lipoprotéines en raison d'un apport insuffisant en certains acides aminés essentiels nécessaires à la formation de la partie protéique des lipoprotéines, les apolipoprotéines (apos) [86]. Chez le lapin [87], le cochon d'Inde [15], le rat [88] et le hamster [89], la caséine, mais pas les PS, élève significativement les niveaux de LDL et de VLDL et d'apo $B$. L'effet résulte d'une diminution du 
catabolisme des VLDL et des LDL $[87,90]$. Ainsi, l'accumulation de LDL chez les animaux recevant de la caséine est vraisemblablement le résultat d'une diminution de la captation des LDL par diminution de l'activité ou du nombre de leurs récepteurs membranaires. Il a été montré que la caséine réduit le nombre de récepteurs hépatiques aux lipoprotéines [91, 92] et qu'à l'inverse les PS augmentent l'activité des récepteurs aux LDL [93, 94].

Madani et al. [31] montrent que le contenu en protéines des $\mathrm{HDL}_{2-3}$ est en grand partie indépendant du niveau d'apport en protéines, mais que celui des LDL, et surtout des VLDL, augmente avec le niveau d'apport en caséine ou en PS dans le régime. Les teneurs en apos $B_{100}$ et $B_{48}$ augmentent aussi avec l'apport alimentaire en caséine ou en PS, ce qui est corrélé avec un accroissement de la synthèse des VLDL. De plus, avec des régimes contenant seulement $10 \%$ de caséine ou de PS il n'y a pas d'accumulation de lipides dans le foie de rats, donc l'exportation des lipides n'est pas modifiée, mais le régime à $10 \%$ de caséine diminue le nombre de $\mathrm{HDL}_{2-3}$ par diminution de leur synthèse. Les régimes à 10 et $30 \%$ de PS diminuent les concentrations en apos $\mathrm{Al}$ et $\mathrm{C}$ dans les $\mathrm{HDL}_{2-3}$. Par contre, le niveau d'apport en protéines $(10,20$ et $30 \%)$ ne modifie pas le niveau du cholestérol plasmatique [31]. Okita et Sugano [95] ont aussi montré que le cholestérol plasmatique n'est pas modifié par l'augmentation de la caséine dans le régime mais qu'il diminue avec l'augmentation des PS. Minehira et al. [35] montrent que les PS diminuent la concentration de HDL-cholestérol, mais moins que le cholestérol total, et donc que le rapport HDL-cholestérol/cholestérol total est augmenté, du moins chez les jeunes rats, alors que ce rapport est diminué chez les rats âgés. Ils montrent aussi que les isolats de protéines de petit lait sont aussi hypocholestérolémiants, et même davantage que les PS quand les régimes des rats contiennent $0,5 \%$ de cholestérol.

Protéines végétales et peroxydation lipidique

Chez le rat les PS, comparées à la caséine, diminuent la peroxydation lipidique [55]. Kanazawa et al. [96] mettent en évidence un effet protecteur des PS vis à vis de la peroxydation des lipoprotéines chez des patients souffrant d'une atteinte cérébrovasculaire. Cette action peut provenir des isoflavones associées aux PS qui ont des actions antioxydantes [97]. Cependant, les protéines alimentaires jouent un rôle dans les mécanismes de défense antioxydatifs. La nature et le niveau d'apport en protéines, spécialement leur contenu en acides aminés soufrés, influencent la concentration cellulaire en glutathion $[98,99]$, qui joue un rôle capital dans la détoxication des intermédiaires réactifs fournis par le métabolisme oxydatif [100]. Quand le glutathion hépatique est diminué, les animaux et l'homme sont plus sensibles aux dommages tissulaires entraînés par les radicaux libres [101]. Cependant, peu d'études ont porté sur les effets de différentes protéines sur la peroxydation lipidique. Les PS et la caséine entraînent la même résistance des hématies à l'attaque radicalaire, mais les PS diminuent les TBARS (substances réagissant avec l'acide thiobarbiturique, qui sont des produits d'oxydation) sériques, donc la peroxydation lipidique [31], sans modifier ces substances au niveau du foie [35]. Cela peut provenir de la diminution des TG plasmatiques avec le régime $\mathrm{PS}$, en effet, il a été montré une corrélation positive entre l'importance de la peroxydation des lipides plasmatiques et les teneurs en TG du plasma chez l'homme [102]. 
Protéines végétales et tissu adipeux

Les PS sont susceptibles de réduire le dépôt de graisses chez le rat [103, 104]. Ceci serait dû au faible rapport lysine/arginine chez les PS, qui diminue la concentration en insuline, augmente la sensibilité à l'insuline des tissus, la tolérance au glucose et diminue la lipogenèse par suppression de l'expression du gène de l'enzyme lipogénique, comme cela a été montré chez des rats génétiquement obèses [105]. Le niveau d'insuline post-prandial est aussi diminué chez l'homme consommant des PS par rapport à la caséine. L'augmentation de la sensibilité à l'insuline peut aussi diminuer le cholestérol en diminuant sa synthèse hépatique et la sécrétion de VLDL riches en TG. Les PS diminuent les TG du plasma et du foie car elles inhibent la conversion de la thyroxine en triiodothyronine, ce qui diminue la synthèse des acides gras et augmente la lipolyse et la libération de noradrénaline qui possède une activité lipolytique [106].

Malnutrition protéique et métabolisme des lipides et des lipoprotéines

La malnutrition protéique ( $2 \%$ caséine versus $20 \%$ caséine) entraîne une forte diminution des TG circulants qui est corrélée avec une accumulation de ces lipides dans le foie [81, 107]. La réduction de la synthèse des apos de VLDL par le foie est responsable de cette stéatose hépatique, les TG et les esters de cholestérol synthétisés par le foie sont exportés en plus faible quantité [107]. La carence alimentaire, soit en protéines, soit en AGE induit une stéatose hépatique, et un faible apport en protéines intensifie les symptômes d'une carence en AGE [108-111]. L'existence d'une stéatose hépatique et la ressemblance des lésions de la peau avec celles observées dans la carence expérimentale en AGE peuvent toutes les deux être présentes dans la malnutrition chronique [2, 113-115]. Chez l'homme adulte souffrant de malnutrition protéino-énergétique une carence en AGE a été décrite [2]. La carence en AGE peut précipiter le kwashiorkor (malnutrition protéique), aggravant les effets de l'insuffisance d'apport en protéines chez les enfants malnutris et entraînant les lésions cutanées [113]. La carence en AGE perturbe aussi le métabolisme des protéines, elle diminue la rétention azotée [116].

Chez des enfants, la stéatose hépatique est telle parfois que les triglycérides peuvent participer à $50 \%$ du poids du foie. Hill et Holman [111] ont rapporté les effets de différents niveaux d'apport en protéines (5 à $40 \%$ ) sur la disponibilité en AGE chez des rats recevant de l'huile de noix de coco hydrogénée et ils ont noté que les symptômes de la carence en AGE sont amplifiés par des apports faibles en protéines. Chez des enfants souffrant de kwashiorkor, le rapport acides gras insaturés/acides gras saturés diminue dans le plasma, de même que dans les lipides des globules rouges [117].

Chez des enfants nigérians, souffrant d'une malnutrition protéique (MP), les TG, les esters de cholestérol, les phospholipides plasmatiques sont diminués, les VLDL disparaissent et les LDL sont fortement abaissées ainsi que les AGPI [115]. Cette relative carence en AGPI peut contribuer aux symptômes associés à la MP: dermatose desquamante, perte de cheveux, susceptibilité aux 
infections, stéatose hépatique, diminution de la capacité à cicatriser, changements psychomoteurs, retard de croissance qui sont aussi observés dans la carence en AGE. Chez les enfants, la MP entraîne un arrêt de la croissance, avec œdèmes, dermatoses, stéatose hépatique, dépigmentation de la peau et des cheveux et modification de leur texture, une diminution de l'albumine sérique, avec un tissu adipeux sous-cutané proche de la normale. La carence en AGE s'accompagne d'un retard de croissance, d'une dermatose desquamante, d'une augmentation de la perméabilité de la peau accompagnée d'une forte perte hydrique, d'une dépigmentation, d'une stéatose des poumons et du foie [59]. Lors de la MP, le $20: 3 n-6$ est aussi diminué et c'est lui qui est le précurseur des prostaglandines intervenant dans les atteintes de la peau et sur les fonctions des mélanocytes [117].

La MP augmente les teneurs en acides gras saturés et en monoinsaturés et diminue les réserves en AGE. La carence en AGE des enfants nigérians porte surtout sur le $18: 2 n-6$ et ses métabolites. Le $20: 4 n-6$ diminue dans les esters de cholestérol et dans les phospholipides, alors que le rapport $20: 3 n-9 / 20: 4 n-6$ est peu modifié. Ce rapport triène/tétraène est un indicateur de la carence en AGE, sa valeur ne doit pas dépasser 0,2 [118]. De plus, la MP diminue l'absorption des lipides en raison d'une diminution de la production de lipase pancréatique. En outre, l'insuffisance d'apport énergétique peut augmenter l'oxydation des AGPI, ce qui peut majorer la carence en AGE. De plus, la MP diminue l'apport de sélénium, cofacteur de la glutathion peroxydase, et de la vitamine $E$, ce qui peut favoriser la peroxydation lipidique et en particulier celle des AGPI [115]. Chez le rat, un régime pauvre en protéines augmente l'hydrolyse in vitro des érythrocytes soumis à une attaque radicalaire [119], cela provient d'une baisse des activités des enzymes hépatiques antioxydantes (par exemple la glutathion peroxydase et les superoxyde dismutases) [120, 121]. Avec un faible apport en protéines (10\% du régime) les TBARS sanguines augmentent et la résistance des hématies diminue. Donc un faible apport de protéines augmente la peroxydation lipidique.

Les signes dermiques de la carence en AGE peuvent aussi être majorés par des apports excessifs en protéines (au-dessus de $30 \%$ ), par contre, dans les phospholipides de foie le rapport triène/tétraène est accru par la MP et diminué avec l'augmentation de l'apport en protéines [111].

Chez des rats recevant des régimes pauvres en caséine ou en PS, l'ajout de méthionine à ces régimes entraîne une plus forte stéatose hépatique, car la teneur en méthionine du régime joue un rôle important dans le métabolisme des lipides, la méthionine favorisant la lipogenèse hépatique [112].

Chez le rat un régime carencé en protéines diminue, tandis qu'un régime riche en protéines augmente la synthèse du $20: 4 n-6$ à partir du $18: 2 n-6[71,73,122]$. Les protéines alimentaires modulent aussi la synthèse des éicosanoïdes. Morita et al [123] montrent que des animaux recevant des régimes faibles en protéines produisent plus de $T X A_{2}$ et moins de $P G I_{2}$ et ont une plus forte agrégabilité plaquettaire que ceux consommant des régimes riches en protéines.

Dans les phospholipides du plasma et du foie, le $18: 2 n-6$ et ses dérivés sont d'autant plus diminués chez les nourrissons que la MP est plus prononcée. La diminution des acides gras en $n-6$ est en partie compensée par l'augmentation des acides gras en n-9 au niveau des globules rouges, ce qui est 
typique d'une carence en AGE [124]. Les mêmes auteurs en carençant des rates gestantes puis lactantes [125] montrent une diminution de l'activité de la Delta6-désaturase chez la mère ce qui diminue la fourniture d'AGPI au fœtus, et le $20: 4 n-6$ diminue dans les phospholipides hépatiques. Cependant, malgré la perturbation du métabolisme du $18: 2 n-6$ chez la mère la proportion de $20: 4 n-6$ reste normale dans le lait. Malgré le faible apport de protéines (5\%) le cerveau paraît relativement protégé, bien que la synthèse de myéline soit perturbée [125] et qu'il existe une diminution des acides gras en $n-3$ et $n-6$ dans les phosphatidylcholines et les phophatidyléthanolamines des cerveaux avec les régimes à 5 et $10 \%$ de protéines [125].

De Tomas et al. [3] montrent que les activités des Delta5-, Delta6- et Delta9-désaturases sont fortement diminuées par la carence protéique, mais que la carence en AGE ne modifie pas significativement les activités des Delta6-et Delta5-désaturases, mais augmente fortement l'activité de la Delta9-désaturase. Dans les phospholipides hépatiques, la MP augmente les teneurs en $16: 0$, $18: 0,18: 1 n-9$ et elle diminue celle du $20: 4 n-6$. Alors que la carence en AGE augmente les teneurs en acides gras monoinsaturés et en $20: 3 n-9$ et diminue celles du $18: 2 n-6$ et du $20: 4 n-6$. Chez le rat la perturbation de la synthèse du $20: 4 n-6$ à partir du $18: 2 n-6$, résultant d'une carence en protéines, a été attribuée à une réduction des activités des Delta5- et Delta6-désaturases [71, 73, 126-130]. Avec un régime à $2 \%$ de caséine comparé à un régime à $20 \%$, l'activité des Delta6-et Delta5-désaturases diminuent de 20 à $30 \%$ leur valeur originelle après 2 jours de carence protéique, mais après 25 jours du régime à $2 \%$ de caséine, l'activité de la Delta6-désaturase revient à $91 \%$ de la valeur témoin, celle de la Delta5-désaturase à $70 \%$, tandis que la Delta9-désaturase est diminuée de 33 et $55 \%$ après 2 et 14 jours du régime à $2 \%$ de caséine [126]. La MP diminue les acides gras en C20 et C22 dans les phospholipides de microsomes, surtout le $20: 4 n-6$, avec une augmentation du $18: 2 \mathrm{n}-6[126]$.

La Delta6n-3-désaturase est plus inhibée avec la MP en présence d'huile de saumon qu'en présence d'huile de noix de coco hydrogénée, et le $20: 5 n-3$ important dans l'huile de saumon peut inhiber la Delta6n-3-désaturation par un phénomène de rétrocontrôle négatif [81].

Lamri et al. [131, 132] montrent que les activités de la lipoprotéine lipase et de la lipase hépatique diminuent rapidement avec la MP, mais comme les quantités de VLDL sont aussi diminuées leur métabolisme reste assuré, elles ne s'accumulent pas. La MP diminue l'incorporation de ${ }^{3} \mathrm{H}$-leucine dans les apos de VLDL [133], réduit la demi-vie des apos de VLDL et augmente leur clairance au niveau des organes périphériques, mais pas au niveau du foie. Les teneurs plasmatiques en acides gras libres sont aussi diminuées par la MP. Par contre, les HDL montrent peu de modifications lors de très sévères MP ( $2 \%$ de caséine ou $5 \%$ de gluten). Ces régimes carencés en protéines augmentent les TG hépatiques, mais diminuent les phospholipides [134, 135], la diminution des phospholipides dans le foie, principaux constituants des membranes, peut correspondre à une diminution de la taille des cellules. Cette diminution porte surtout sur les phosphatidylcholines, car la carence en groupes méthyl (méthionine) perturbe la formation de la choline. La MP diminue les AGE dans les TG et les phospholipides des VLDL. La diminution de l'apo CII par le foie peut expliquer la faible activité de la lipoprotéine lipase dont l'apo Cll est un cofacteur activateur et cela explique que la teneur en TG des VLDL soit augmentée [134]. La MP augmente les teneurs du $22: 5 n-6$ et du $18: 2 n-6$ dans les 
phospholipides de foie, en raison d'une sous-utilisation du $22: 5 n-6$, cependant le rapport $20: 4 n$ $6 / 18: 2 n-6$ reste semblable dans les phopholipides de foie avec le régime équilibré en protéines et les deux régimes carencés.

Bouziane et al. [136] en utilisant diverses huiles (tournesol, soja, noix de coco hydrogénée, saumon) avec des apports de $2 \%$ de caséine montrent que la MP diminue les phospholipides dans le foie et les VLDL, surtout avec l'huile de noix de coco. Le rapport du $20: 3 n-9 / 20: 4 n-6$ atteint des valeurs supérieures à 0,2 dans les phospholipides sériques avec les huiles de coco et de saumon, ce qui signe une carence en AGE [118] avec ces deux huiles lorsque l'apport en protéines est insuffisant. On retrouve les mêmes résultats chez les enfants malnutris [2, 113]. Le rapport acides gras polyinsaturés/saturés est diminué par la MP dans les TG et phospholipides du foie et des VLDL, le rapport le plus élevé est obtenu avec l'huile de saumon [136] et les acides gras en n-6, $18: 2 n-6$ et $20: 4 n-6$ sont diminués dans les phospholipides de foie et de VLDL et la transformation de l'EPA en DHA est accélérée par la MP. Les apos $B$ et $C$, principalement synthétisées par le foie, sont particulièrement diminuées. Comme le $20: 4 n-6$ et le $20: 5 n-3$ sont des précurseurs de prostaglandines, de thromboxanes et de leucotriènes leur diminution avec la MP peut expliquer une partie des symptômes cliniques du kwashiokor [136].

\section{CONCLUSION}

Les protéines végétales, particulièrement la fraction indigeste, diminuent l'absorption intestinale du cholestérol, augmentent l'excrétion fécale des stérols et améliorent le catabolisme des lipoprotéines portant l'essentiel du cholestérol en augmentant l'activité ou le nombre des récepteurs aux LDL. Dans les cellules, les protéines alimentaires modifient le rapport cholestérol/phospholipides et en conséquence la fluidité des membranes microsomales. Les changements de propriétés des membranes microsomales non seulement influencent le métabolisme des acides gras polyinsaturés mais aussi celui du cholestérol. De plus, les protéines alimentaires avec des compositions différentes en acides aminés (par exemple lysine et arginine) peuvent moduler la sécrétion d’hormones (par exemple le glucagon et l'insuline), qui en retour peuvent modifier l'activité des enzymes responsables du métabolisme du cholestérol (par exemple la HMG-CoA réductase) et du métabolismes des acides gras polyinsaturés (par exemple les Delta5- et Delta6-désaturases).

Les résultats dépendent de l'espèce utilisée, de la présence ou non de composés non-protéiques associés aux protéines, donc de leur degré de pureté, de l'addition ou non de cholestérol et de sa quantité dans les régimes.

La malnutrition protéique augmente les besoins en AGE, ce qui laisse supposer qu'une partie des symptômes associés à la MP relève de l'insuffisance d'apport en AGE. Cela suppose que les régimes de récupération nutritionnelle doivent non seulement satisfaire les besoins en protéines mais aussi apporter les acides gras indispensables en quantité suffisante. La carence d'apport en protéines diminue la synthèse du $20: 4 n-6$ à partir du $18: 2 n-6$, par diminution des activités des Delta5 et 
Delta6-désaturases. Les protéines peuvent donc moduler la synthèse des eicosanoïdes et par exemple un faible apport en protéines produit plus de thromboxane $A_{2}$ et moins de prostaglandine $I_{2}$, ce qui peut favoriser les maladies cardiovasculaires.

\section{REFERENCES}

Sanders TA, Rana SK. Comparison of the metabolism of linoleic and linolenic acids in the fetal rat. Ann Nutr Metab 1987 ; 31 : 349-53.

Holman RT, Johnson SB, Mercuri C, Itarte HJ, Rodrigo MA, De Tomas ME. Essential fatty acid deficiency in malnourished children. Am J Clin Nutr 1981 ; 34 : 1534-9.

De Tomas ME, Mercuri O, Rodrigo A. Effects of dietary protein and EFA deficiency on liver Delta5-, Delta6- and Delta9-desaturase activities in the early developing rat. J Nutr $1979 ; 110$ : 595-9.

Carroll K, Hamilton RMG. Effects of dietary protein and carbohydrate on plasma cholesterol levels in relation to atherosclerosis. J Food Sci $1975 ; 40: 18-23$.

Kritchevsky D. Vegetable protein and atherosclerosis. J Am Oil Chem Soc 1979 ; 56 : 135-40.

Sakono M, Yoshida K, Yahiro M. Combined effects of dietary protein and fat on lipid metabolism in rats. J Nutr Sci Vitaminol 1993 ; 39 : 335-43.

Lovati MR, West CE, Sirtori CR, Beynen AC. Dietary animal proteins and cholesterol metabolism in rabbits. Br J Nutr $1990 ; 64: 473-85$.

van der Meer R, Beynen AC (1987) Species-dependent responses of serum cholesterol to dietary proteins. J Am Oil Chem Soc ; 64 : 1172-7.

Choi YS, Ikeda I, Sugano M. Dietary fats modulate age-dependent effects of dietary proteins on cholesterol metabolism. J Nutr Sci Vitaminol 1990 ; 36 (Suppl) S181-4.

Gatchalian YM, Arimura Y, Ochiai E, Yamada K, Sugano M. Soybean protein lowers serum cholesterol level in hamsters: Effect of debittered undigested fraction. Nutrition $1997 ; 13: 633-9$.

Carroll KK, Kurowska EM. Soy consumption and cholesterol reduction: Review of animal and human studies. J Nutr 1995 ; (Suppl), 125 : 594S-7. 
Park MS, Kudchodkar BJ, Liepa GU. Effects of dietary animal and plant proteins on the cholesterol metabolism in immature and mature rats. J Nutr 1987 ; $117: 30-5$.

Forsythe WA, Miller ER, Hill GM, Romsos DR, Simpson RC. Effects of dietary protein and fat sources on plasma cholesterol parameters, LCAT activity and amino acids levels and on tissue lipid content of growing pigs. J Nutr $1980 ; 110: 2467-79$.

Park MS, Liepa GU. Effects of dietary protein and amino acids on the metabolism of cholesterolcarrying lipoproteins in rats. J Nutr $1982 ; 112$ : 1892-8.

Atwal AS, Kubow S, Wolynetz MS. Effects of protein source and amino acid supplementation on plasma cholesterol in Guinea Pigs. Internat J Vit Nutr Res 1997 ; 67 : 192-5.

Carroll KK. Review of clinical studies on cholesterol-lowering response to soy protein. J Am Dietetic Assoc $1991 ; 91: 820-7$.

Meinertz H, Nilausen K, Faergemen O. Effect of dietary protein on plasma lipoprotein levels in normal subjects: Interaction with dietary cholesterol. J Nutr Sci Vitaminol 1990 ; 36 : S157-64.

Kritchevsky D. Dietary protein and experimental atherosclerosis. Ann N. Y. Acad Sci $1993 ; 676: 180-$ 7.

Sirtori CR, Even R, Lovati MR. Soybean protein diet and plasma cholesterol: from therapy to molecular mechanism. Ann N. Y. Acad Sci $1993 ; 676: 188-201$.

Sugano M, Koba K. Dietary protein and lipid metabolism: a multifunctional effect. Ann N.Y. Acad Sci $1993 ; 676: 215-22$.

Gaddi A, Descovich GC, Noseda G. Hypercholesterolaemia treated by soybean protein diet. Arch Dis Child $1987 ; 62: 274-8$

Kritchevsky D. Dietary protein, cholesterol and atherosclerosis: A review of the early history. J Nutr $1995 ; 125$ : 589S-93.

Nagata $\mathrm{Y}$, Ishiwaki N, Sugano M. Studies on the mechanism of the antihypercholesterolemia action of soy protein and soy protein-type amino acid mixtures in relation to their casein counterparts in rats. J Nutr $1982 ; 112: 1614-25$. 
Beynen A. Comparison of the mechanisms proposed to explain the hypocholesterolemic effect of soybean protein versus casein in experimental animals. J Nutr Sci Vitaminol $1990 ; 36$ : S87-93.

Vahouny GV, Adamson I, Chalcarz W, Satchithanandam S, Muesing R, Klurfeld DM, et al. Effects of casein and soybean protein on hepatic and serum lipids and lipoprotein lipid distributions in the rat. Atherosclerosis $1985 ; 56: 127-37$.

Iwami K, Sakakibara K, Ibuki F. Involvement of post-digestion hydrophobic peptides in plasma cholesterol-lowering effect of dietary plant protein. Agric Biol Chem 1986 ; 50 : 1217-22.

Sugano $M$, Yamada $\mathrm{Y}$, Yoshida $\mathrm{Y}$, Hashimoto $\mathrm{T}$, Matsuo $\mathrm{T}$, Kimoto $\mathrm{M}$, . The hypocholesterolemic action of the undigested fraction of soybean protein in rats. Atherosclerosis $1988 ; 72: 115-22$.

Sugano M, Goto S, Yamada Y, Yoshida K, Hashimoto T, Matsuo T, Kimoto M. Cholesterol lowering activity of various undigested fractions of soybean protein in rats. J Nutr $1990 ; 120$ : 977-85.

Ogawa T, Gatchalian-Yee M, Sugano M, Kimoto M, Matsuo T, Hashimoto Y. Hypocholesterolemic effect of undigested fraction of soybean protein in rats fed no cholesterol. Biosci Biotech Biochem $1992 ; 11: 1845-8$.

Younes $\mathrm{H}$, Levrat MA, Demigne C, Remesy C. Resistant starch is more effective than cholestyramine as a lipid-lowering agent in the rat. Lipids $1995 ; 30: 847-53$.

Madani S, Prost J, Belleville J. Dietary protein level and origin (casein and highly purified soybean protein) affect hepatic storage, plasma lipid transport, and antioxidative defense status in the rat. Nutrition $2000 ; 16: 368-75$.

Madani S, Lopez S, Blond JP, Prost J, Belleville J.. Highly purified soybean protein is not hypocholesterolemic in rats but stimulates cholesterol synthesis and excretion and reduces polyunsaturated fatty acid biosynthesis. J Nutr $1998 ; 128: 1084-91$.

Choi YS, Goto S, Ikeda I, Sugano M. Interaction of dietary protein, cholesterol and age on lipid metabolism of the rat. Br J Nutr $1989 ; 61: 531-43$.

Lee JH, Ikeda I, Sugano M. Dietary cholesterol influences on various lipid indices and eicosanoid production in rats fed dietary fat desirable for the protection of ischemic disease. J Nutr Sci Vitaminol $1991 ; 37$ : 389-99. 
Minehira K, Inoue S, Nonaka M, Osada K, Yamada K, Sugano M. Effects of dietary protein type on oxidized cholesterol-induced alteration in age-related modulation of lipid metabolism and indices of immune function in rats. Biochim Biophys Acta $2000 ; 1483: 141-53$.

Nagaoka S, Kanamaru Y, Kuzuya Y Effects of whey protein and casein on the plasma and liver lipids in rats. Agric Biol Chem $1991 ; 55$ : 813-8.

Balmir F, Staack R, Jeffrey E, Jimenez M, Wang L, Potter S. An extract of soy flour influences serum cholesterol and thyroid hormones in rats and hamsters. J Nutr $1996 ; 126$ : 3046-53.

Tasker TE, Potter SM. Effects of dietary protein source on plasma lipids, HMG-CoA reductase activity, and hepatic glutathione levels in gerbils. J Nutr Biochem $1993 ; 4: 458-62$.

Potter SM. Overview of proposed mechanisms for the hypocholesterolemic effect of soy. J Nutr $1995 ; 125$ : 606S-11.

Tsai P, Huang P. Effects of isoflavones containing soy protein isolate compared with fish protein on serum lipids and susceptibility of low density lipoprotein and liver lipids to in vitro oxidation in hamsters. J Nutr Biochem $1999 ; 10$ : 631-7.

Anthony MS, Clarkson TB, Hughes CL, Morgan TM, Burke GL. Soybean isoflavones improve cardiovascular risk factors without affecting the reproductive system of peripubertal rhesus monkeys. J Nutr $1996 ; 126$ : 43-50.

Saeki S, Kiriyama S. Some evidence excluding the possibility that rat plasma cholesterol is regulated by the modification of enterohepatic circulation of steroid. In: Dietary Protein, Cholesterol Metabolism and Atherosclerosis, Monographs on Atherosclerosis (Sugano M et Beynen AC, eds.) ; 1990: vol. 16, pp. 71-84. Karger, Basel, Switzerland.

Huff MW, Hamilton RMG, Carroll KK. Plasma cholesterol in rabbits fed low fat, cholesterol free, semipurified diets: Effects of dietary protein, protein hydrolysates and amino acid mixtures. Atherosclerosis $1977 ; 28: 187-95$.

FAO. Amino-acids content of foods and biological data on proteins. Food Policy and Food Sci Service, Nutrition Division, FAO, Rome ; 1970 : No. 24, pp. 56-57 and 132-3. 
Sugiyama K, Ohkawa S, Muramatsu K. Relationship between amino acid composition of diet and plasma cholesterol level in growing rats fed a high cholesterol diet. J Nutr Sci Vitaminol 1986 ; 32 : 413-23.

Sugiyama K, Yamakawa A, Kumazawa A, Saeki S. Methionine content of dietary proteins affects the molecular species composition of plasma phosphatidylcholine in rats fed a cholesterol-free diet. J Nutr $1997 ; 127: 600-7$.

Sautier C, Dieng K, Flament C, Doucet C, Suquet JP, Lemonnier D. Effect of whey protein, casein, soybean and sunflower proteins on the serum, tissue and fecal steroids in rats. Br J Nutr $1983 ; 43$ : 313-9.

Sautier C, Flament C, Doucet C, Suquet JP. Effect of eight dietary proteins and their amino acid content on serum, hepatic and fecal steroids in the rat. Nutr Rep Int $1986 ; 34$ : 1051-61.

Muramatsu K, Sugiyama K. Relationship between amino acid composition of dietary protein and plasma cholesterol level in rats. In: Dietary Proteins, Cholesterol metabolism and Atherosclerosis (Sugano M and Beynen AC, eds.) Monogr Atheroscler 1990 ; Vol 16, pp 97-109. Karger, Basel.

Morita T, Oh-Hashi A, Taeki K, Ikai M, Kasaoka S, Kiriyama S. Cholesterol-lowering effects of soybean, potato, and rice proteins depend on their low methionine contents in rats fed a cholesterol-free purified diet. J Nutr $1997 ; 127: 470-7$.

Huang YS, Koba K, Horrobin DF, Sugano M. Interrelationship between dietary protein, cholesterol and n-6 polyunsaturated fatty acid metabolism. Prog Lipid Res $1993 ; 32$ : 123-37.

Sugano M, Ishiwaki N, Nakashima K. Dietary protein-dependent modification of serum cholesterol level in rats. Ann Nutr Metab $1984 ; 28: 192-99$.

Beg ZH, Stonik JA, Brewer HB. In vitro and in vivo phosphorylation of rat liver 3-hydroxy-3methylglutaryl coenzyme A reductase and its modulation by glucagon. J Biol Chem $1980 ; 255: 8541$ 5.

Sirtori CR, Galli G, Lovati MR, Carrara P, Bosisio E, Kienle MG. Effects of dietary proteins on the regulation of liver lipoprotein receptors in rats. J Nutr $1984 ; 114: 1493-500$.

Sugano $\mathrm{M}$, Ishiwaki N, Nagata $\mathrm{Y}$, Amaizumi K. Effect of arginine and lysine addition to casein and soya-bean protein on serum lipids, apolipoproteins, insulin and glucagon in rats. Br J Nutr $1982 ; 48$ : 211-21. 
Beynen AC, Katan MB. Impact of dietary cholesterol and fatty acids on serum lipids and lipoproteins in man, in The Role of Fats in Human Nutrition, $2^{\text {nd }}$ Ed. by Vergroesen, $\mathrm{AJ}$ and Crawford $\mathrm{M}$, Academic Press Inc., San Diego ; 1989 : pp. 152-235.

Hornstra G. The significance of fish and fish-oil enriched food for prevention and therapy of ischaemic cardiovascular disease, in The Role of Fats in Human Nutrition, $2^{\text {nd }}$ Ed., ed. by Vergroesen AJ, and Crawford M, Academic Press Inc. San Diego ; 1989 : pp 152-235.

Kinsella JE. Effects of polyunsaturated fatty acids on factors related to cardiovascular disease. Am J Cardiol $1987 ; 60: 23 \mathrm{G}-32$.

Brenner RR. Factors influencing fatty acid chain elongation and desaturation, in The Role of Fats in Human Nutrition ; $2^{\text {nd }}$ Ed., ed. by Vergroesen, AJ, and Crawford M, Academic Press Inc. San Diego ; 1989 : pp. 237-86.

Kumamoto T, Ide T. Comparative effects of alpha- and gamma-linolenic acids on rat liver fatty acid oxidation. Lipids $1998 ; 33: 647-54$.

Inui K, Fukuta Y, Ikeda A, Kameda H, Kokuba Y, Sato M. The effect of (-linolenic acid-rich emulsion on fatty acid metabolism and leukotriene generation of the colon in a rat model with inflammatory bowel disease. Ann Nutr Metab $1996 ; 40: 175-82$.

Goetzl EJ Oxygenation products of arachidonic acid as mediators of hypersensitivity and inflammation. Med Clin North Am $1981 ; 65$ : 809-28.

Kaku S, Yunoki S, Ohkura K, Sugano M, Nonaka M, Tachibana H, Yamada K. Interactions of dietary fats and proteins on fatty acid composition of immune cells and LTB4 production by peritoneal exudate cells of rats. Biosci Biotechnol Biochem $2001 ; 65: 315-21$.

Holman RT (1964). Nutritional and metabolic interrelationships between fatty acids. Fed Proc ; 23 : 1062-7.

Iritani N, Komiya M, Fukuda H, Sugimoto T. Lipogenic enzyme gene expression is quickly suppressed in rats by a small amount of exogenous polyunsaturated fatty acids. J Nutr $1998 ; 128$ : 967-72.

Huang YS, Cunnane SC, Horrobin DF. Effect of different dietary proteins on plasma and liver fatty acid compositions in growing rats. Proc Soc Exp Biol Med $1986 ; 181$ : 399-403. 
Koba K, Wakamatsu K, Obata K, Sugano M. Effects of dietary proteins on linoleic desaturation and membrane fluidity in rat liver microsomes. Lipids $1993 ; 28: 457-64$.

Iritani N, Sugimoto T, Fukuda H, Komiya M, Ikeda H. Dietary soybean protein increases insulin receptor gene expression in wistar fatty rats when dietary polyunsaturated fatty acid level is low. J Nutr $1997 ; 127$ : 1077-83.

Brenner RR Nutritional and hormonal factors influencing desaturation of essential fatty acids. Progr Lipid Res $1981 ; 20: 41-7$.

Inkpen CA, Harris RR, Quackenbush FQ. Differential responses to fasting and subsequent feeding by microsomal systems of rat liver: Delta6- and Delta9-desaturation of fatty acids. J Lipid Res $1969 ; 10$ : $277-82$

Peluffo RO, de Gommez Dumm NT, de Alaniz MJ, Brenner RR. Effect of protein and insulin on linoleic acid desaturation of normal and diabetic rats. J Nutr $1971 ; 101: 1075-84$.

Peluffo RO, Nervi AB, Gonzalez MS, Brenner RR. Effect of different amino acid diets on Delta5-, Delta6- and Delta9-desaturases. Lipids $1984 ; 19$ : 154-7.

Peluffo RO, Brenner RR. Influence of dietary protein on delta6- and Delta9- desaturation of fatty acids in rats of different ages and in different seasons. J Nutr ; 104 : 894-900.

Brenner RR (1981). Nutritional and hormonal factors influencing desaturation of essential fatty acids. Progr Lipid Res $1974 ; 20$ : 41-7.

Huang YS, Mitchell J, Mc Adoo K, Horrobin DF. Effect of lysine supplementation to wheat gluten on plasma and liver cholesterol and fatty acids in growing rats. Biochem Arch $1987 ; 3$ : 285-93.

Koba K, Abe K, Sugano M. Effects of amino acid composition of dietary protein on linoleic acid desaturation in rats. Agric Biol Chem 1990 ; 54 : 2711-17.

Koba K, Sugano M. Dietary protein influences polyunsaturated fatty acid patterns of rat tissue lipids. J Nutr Sci Vitaminol $1990 ; 36$ (Suppl.) : S173-6.

Sugano M, Ishida T, Koba K. Protein-fat interaction on serum cholesterol level, fatty acid desaturation and eicosanoid production in rats, J Nutr $1988 ; 118: 548-54$. 
Imaizumi K, Abe K, Kuroiwa C, Sugano M.. Fat containing stearic acid increases fecal neutral steroid excretion and catabolism of low density lipoproteins without affecting plasma cholesterol concentration in hamsters fed a cholesterol-containing diet. J Nutr $1993 ; 10: 1693-702$.

Choi YS, Ikeda I, Sugano M. The combined effect of dietary proteins and fish oil on cholesterol metabolism in rats of different ages. Lipids $1989 ; 24: 506-10$.

Bouziane M, Prost J, Belleville J. Changes in fatty acid compositions of total serum and lipoprotein particles, in growing rats given protein-deficient diets with hydrogenated coconut or salmon oils as fat sources. Br J Nutr $1994 ; 71: 375-87$.

Noguchi A, Takita T, Suzuki K, Nakamura K, Innami S. Effects of casein and soy-protein on (-linoleic acid metabolism in rats. J Nutr Sci Vitaminol $1992 ; 38: 579-91$.

Ikeda A, Koba K, Sugano M. Impact of dietary protein on polyunsaturated fatty acid desaturation in rats fed diets rich in alpha-linolenic acid. Biosci Biotech Biochem 1993 ; 57 : 61-4.

Terasawa F, Hirano Y, Wada M, Takita T, Nakamura K, Innami S. Effects of dietary casein and soyprotein on metabolic conversion of eicosapentaenoic acid to docosahexaenoic acid in the liver of rat. J Nutr Sci Vitaminol $1994 ; 40$ : 353-62.

Ulmann L, Bouziane M, Mimouni V, Belleville J, Poisson Jp. Relationship between rat liver microsomal Delta6-and Delta5-desaturase activities and fatty acid composition: Comparative effects of coconut and salmon oils during protein restriction. J Nutr Biochem $1992 ; 3$ : 188-93.

Sanchez A, Filler KM, Hubbard RW, Shavlik GW. Dietary protein control of serum cholesterol by insulin and glucagon. Monogr Atheroscler $1988 ; 16: 139-47$.

Samman S, Khosla P, Carroll KK. Effects of dietary casein and soy protein on metabolism of radiolabelled low density apolipoprotein B in rabbits. Lipids $1989 ; 24: 169-72$.

Eklund A, Sjoblom L. Effects of the source of dietary protein on serum lower density lipoprotein (VLDL + LDL) and tocopherol levels in female rats. J Nutr $1980 ; 110: 2321-35$.

Terpstra AH, Holmes JC, Nicolosi RJ. The hypocholesterolemic effect of dietary soybean protein vs casein in hamsters fed cholesterol-free or cholesterol-enriched semipurified diets. J Nutr $1991 ; 121$ : 944-7. 
Khosla P, Samman S, Carroll KK, Huff MW. Turnover of ${ }^{125}$ I-VLDL and ${ }^{131}$ I-LDL apolipoprotein B in rabbits fed diets containing casein or soy protein. Bioch Biophys Acta $1989 ; 1002$ : 157-63.

Chao Y, Yamin TT, Alberts AW (1989). Cholestyramine on low density lipoprotein binding sites on liver membranes from rabbits with endogenous hypercholesterolemia induced by a wheat starch-casein diet. J Biol Chem ; 257 : 3623-7.

Scholz KE, Beynen AC, West CE. Comparison between the hypercholesterolaemia in rabbits induced by semipurified diets containing either cholesterol or casein. Atherosclerosis $1982 ; 44$ : 85-97.

Loukas S, Varoucha D, Zioudrou C, Streaty RA, Klee WA. Opioid activities and structures of alphacasein-derived exorphines. Biochemistry $1983 ; 22: 4567-73$.

Sirtori CR, Agardi E, Conti F, Mantero O, Gatti E. Soybean-protein diet in the treatment of type II hyperlipoproteinaemia. Lancet 1977 ; i : 275-7.

Okida T, Sugano M. Effects of dietary protein levels and cholesterol on tissue lipids of rats. J Nutr Sci Vitaminol $1990 ; 36$ : S151-6.

Kanazawa T, Osanai T, Zhang XS, Uemura T, Yin XZ, Onodera K, Oike Y, Ohkubo K. Protective effects of soy protein on the peroxidability of lipoproteins in cerebrovascular diseases. J Nutr $1995 ; 125$ : 639S-46.

Esaki H, Onozaki H, Morimatsu Y, Kawakishi S, Osawa T. Potent antioxidative isoflavones isolated from soybeans fermented with aspergillus saitoi. Biosci Biotechnol Biochem 1998 ; 62 : 740-6.

Hum S, Koski KG, Hoffer LJ Varied protein intake alters glutathione metabolism in rats. J Nutr 1992 ; $122: 2010-8$.

Moundras C, Remesy C, Levrat MA, Demigne C. Methionine deficiency in rats fed soy protein induces hypercholesterolemia and potentiates lipoprotein susceptibility to peroxidation. Metabolism 1995 ; $9: 1146-52$.

Kaplowitz N, Aw TY, Ookhtens M. The regulation of hepatic glutathione. Annu Rev Pharmacol Toxicol $1985 ; 25: 715-44$

Deneke SM, Gershoff SN, Fanburg BL. Potentiation of oxygen toxicity in rats by dietary protein or amino acids deficiency. J Appl Physiol Respir Environ Exer Physio 1983 ; 54 : 147-51. 
Ledwozyw A, Michalak J, Stepien A, Kadziolka A. The relationship between plasma triglycerides, cholesterol, total lipids and lipid peroxidation products during human atherosclerosis. Clin Chim Acta $1986 ; 155: 275-83$

Hurley $C$, Richard D, Deshaies $Y$, Jacques $H$. Soy protein isolate in the presence of cornstarch reduces body fat gains in rats. Can J Physiol Pharm $1998 ; 76: 1000-7$.

Baba N, Radwan H, Itallie T. Effects of casein versus soy protein diets on body composition and serum lipid levels in adult rats. Nutr Res $1992 ; 12: 279-86$.

Lavigne $\mathrm{C}$, Marette A, Jacques $\mathrm{H}$. Cod and soy proteins compared with casein improve glucose tolerance and insulin sensitivity in rats. Am J Physiol Endocrinol Metab $2000 ; 278$ : E491-500.

Iritani N, Hosomi H, Fukuda H, Tada K, Ikeda H. Soybean protein suppresses hepatic lipogenic enzyme gene expression in wistar fatty rats. J Nutr $1996 ; 126: 380-8$.

Meghelli-Bouchenak M, Boquillon M, Belleville J. Time-course of changes in rat serum apolipoproteins during the consumption of different low protein diets followed by a balanced diet. J Nutr $1987 ; 117: 641-9$.

Alfin-Slater RB, Aftergood L, Wells AF, Deuel HJ. Arch Biochem Biophys $1954 ; 52$ : 180-5.

Fukazawa T, Privett OS, Takahashi Y. Effect of EFA deficiency on lipid transport from liver. Lipids $1971 ; 6: 388-93$.

Gerson T, Wong HN. The effect of protein deficiency on some rat liver lipid metabolic enzymes and CoA. Lipids $1978 ; 13: 446-50$.

Hill EG, Holman RT. Effect of dietary protein level upon essential fatty acid (EFA) deficiency. J Nutr $1980 ; 110: 1057-60$.

Ponzio de Azevedo AM, Azzolin IR, Perry ML. Effect of methionine supplementation to a low-soybean protein diet on liver lipid metabolism. Ann Nutr Metab 1994 ; 38 : 301-6.

Naismith DJ. Kwashiorkor in western Nigeria. A study of traditional weaning food, with particular reference to energy and linoleic acid. Br J Nutr $1973 ; 30: 567-76$. 
Nichols BL, Alvarado J, Rodriguez S, Hazlewood CF, Viteri F. Implications of electrolyte, water, and nitrogen losses during recovery from protein-calorie malnutrition. J Pediatr $1974 ; 84$ : 759-68.

Koletzko B, Abiodun PO, Laryea MD, Bremer HJ. Fatty acid composition of plasma lipids in Nigerian children with protein-energy malnutrition. Eur J Pediatr $1986 ; 145: 109-15$.

Henry CJ, Ghusain-Choueiri A. Protein utilization, growth and survival in essential-fatty-acid-deficient rats. Br J Nutr $1996 ; 75: 237-48$.

Wolff JA, Margolis S, Bujdoso-Wolff K, Matusick E, MacLean WC. Plasma and red blood cell fatty acid composition in children with protein-calorie malnutrition. Pediat Res $1984 ; 18$ : 162-7.

Holman RT. Essential fatty acid deficiency in humans. CRC Handbook Series in Nutrition and Food $1978 ; 3,335-53$.

Huang CJ, Cheng NS, Lu VR. Effects of deteriorated frying oil and dietary protein levels on liver microsomal enzymes in rats. J Am Oil Chem Soc $1988 ; 65: 1796-803$.

Huang CJ, Fwu ML. Degree of protein deficiency affects the extent of the depression of the antioxidative enzyme activities and the enhancement of tissue lipid peroxidation in rats. J Nutr $1993 ; 123: 803-10$.

Moundras C, Demigne C, Morand C, Levrat MA, Remesy C. Lipid metabolism and lipoprotein susceptibility to peroxidation are affected by a protein-deficient diet in the rat. Nutr Res $1997 ; 17$ : 125-35.

Gerson T. Comparison of the effects of dietary protein and lipid deprivation on lipid composition of liver membranes in rats. J Nutr $1974 ; 104: 701-9$.

Morita I, Takahashi R, Ebisawa H, Fujita Y, Murota S. Effect of dietary protein level on platelet aggregation in rat. Prostaglandins Leuko Med $1985 ; 18: 143-9$.

Marin MC, De Tomas ME, Mercuri O, Fernandez A De Serres CT. Interrelationship between proteinenergy malnutrition and essential fatty acid deficiency in nursing infants. Am J Clin Nutr $1991 ; 53$ : 466-8. 
Marin MC, De Tomas ME, Serres C, Mercuri O. Protein-energy malnutrition during gestation and lactation in rats affects growth rate, brain development and essential fatty acid metabolism. J Nutr $1995 ; 125: 1017-24$.

Narce M, Poisson JP, Belleville J, Chanussot B. Time-course effects of protein malnutrition on hepatic fatty acid Delta6-and Delta5-desaturation in the growing rat. Br J Nutr $1988 ; 60: 389-402$.

Mercuri O, De Tomas ME, Itarte H. Prenatal protein depletion and Delta9-, Delta6-and Delta5desaturases in the rat. Lipids $1979 ; 14: 822-5$.

Lindholm M, Eklund A. The effects of dietary protein on the fatty acid composition and delta 6 desaturase activity of rat hepatic microsomes. Lipids $1991 ; 26: 107-19$.

Horgan DJ, Masters CJ. Fatty acid components of ovine tissue lipids, and the response to prolonged protein depletion. Aust J Biol Sci $1963 ; 16$ : 905-15.

De Gomez Dumm IN, Peluffo RO, Brenner RR. Comparative effect of a protein diet on the desaturation, elongation and simultaneous desaturation and elongation of linoleic acid. Lipids 1972 ; $7: 590-5$.

Lamri MY, Meghelli-Bouchenak M, Boualga A, Belleville J, Prost J. Time course of changes in rat serum lecithin-cholesterol acyl transferase activity and high-density-lipoprotein composition during the consumption of two different low-protein diets followed by a balanced diet. Nutrition $1995 ; 11$ : 444-9.

Lamri MY, Meghelli-Bouchenak M, Boualga A, Belleville J, Prost J. Rat plasma VLDL composition and concentration and hepatic lipase and lipoprotein lipase activities are impaired during two types of protein malnutrition and unaffected by balanced refeeding. J Nutr $1995 ; 125: 2425-34$.

Meghelli-Bouchenak M, Belleville J. Metabolism of serum VLDL-, LDL-, and HDL-apoproteins in rats on two different low protein diets. Nutr Res $1991 ; 11: 575-86$.

Meghelli-Bouchenak M, Belleville J, Boquillon M. Hepatic steatosis and serum very low density lipoproteins during two types of protein malnutrition followed by balanced refeeding. Nutrition $1989 ; 5$ : 321-9.

Meghelli-Bouchenak M, Boquillon M, Belleville J. Serum lipoprotein composition and amounts during the consumption of two different low-protein diets followed by a balanced diet. Nutr Rep Int 1989 ; 39 : 323-43. 
Bouziane M, Prost J, Belleville J. Dietary protein deficiency affects $n-3$ and $n-6$ polyunsaturated fatty acids hepatic storage and very low density lipoprotein transport in rats on different diets. Lipids

$1994 ; 29$ : 265-72.

Illustrations

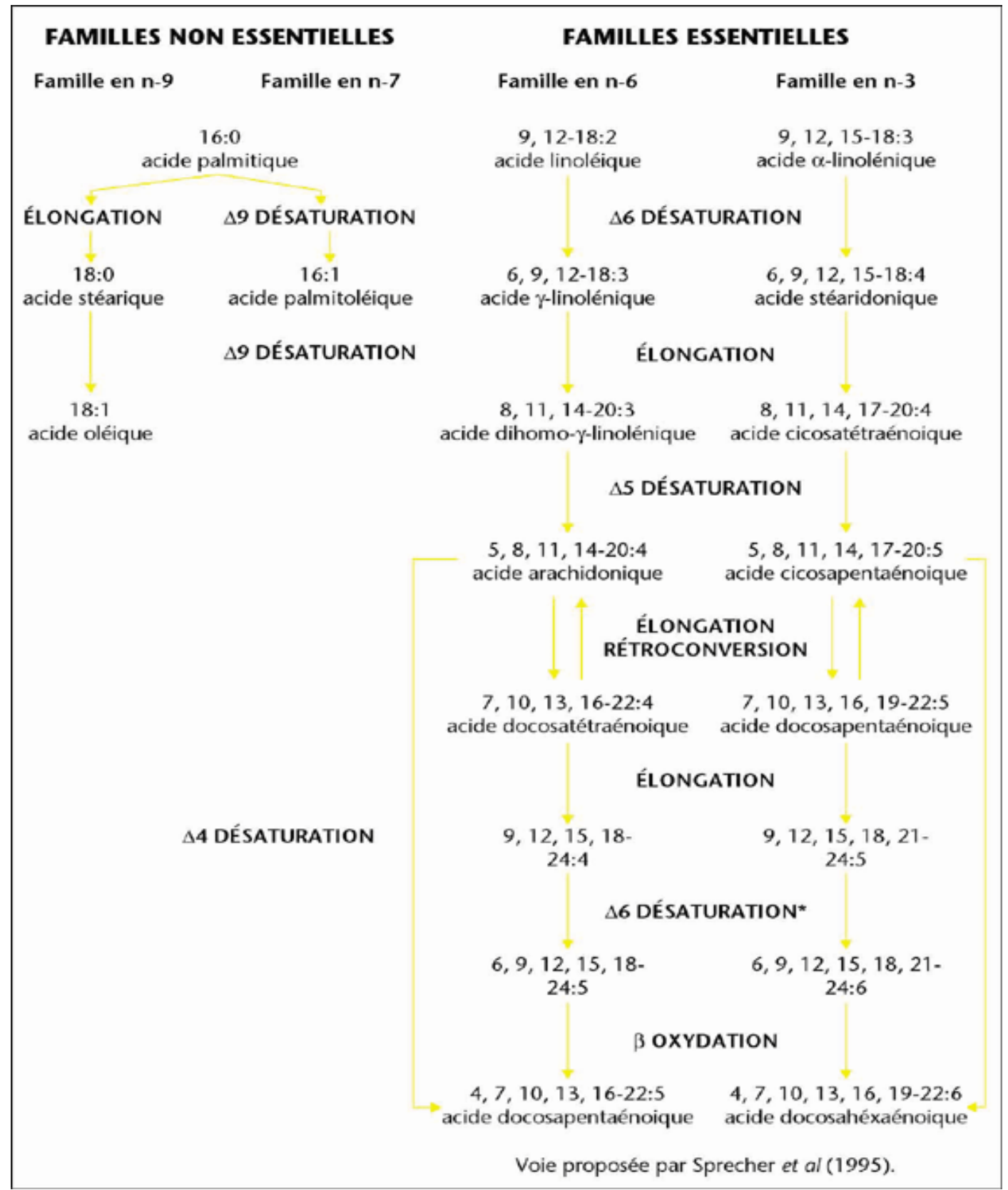

Figure 1. Biosynthèse des acides gras monoinsaturés et polyinsaturés à longue chaîne. 


\begin{tabular}{|c|c|c|c|c|c|}
\hline AC & Endoperoxydes & Prostaglantines & Thromboxanes & Prostacyclines & Leucotriènes \\
\hline $20: 3 n-6$ & $P G H_{1}$ & $\begin{array}{l}P G D_{1} \\
P G E_{1} \\
P G F_{1} a\end{array}$ & $\mathrm{TXA}_{1}$ & $\mathrm{PCI}_{1}$ & \\
\hline $20: 4 n-6$ & $\begin{array}{l}\mathrm{PG} \mathrm{G}_{2} \\
\mathrm{PG} \mathrm{H}_{2}\end{array}$ & $\begin{array}{l}P G D_{2} \\
P G E_{2} \\
P G F_{2} \alpha\end{array}$ & $\begin{array}{l}\mathrm{TXA}_{1} \\
\mathrm{TXB}_{2}\end{array}$ & $\mathrm{PCl}_{2}$ & $\begin{array}{l}\mathrm{LTA}_{4} \\
\mathrm{LTB}_{4} \\
\mathrm{LTC}_{4}\end{array}$ \\
\hline $20: 5 n-3$ & $\begin{array}{l}P^{P G ~ G} \\
P G H_{3}\end{array}$ & $\begin{array}{l}P G D_{3} \\
P^{P G} E_{3} \\
P G F_{3} \alpha\end{array}$ & $\begin{array}{l}\mathrm{TXA}_{3} \\
\mathrm{TXB}_{3}\end{array}$ & $\mathrm{PGI}_{3}$ & $\begin{array}{l}\text { LTA }_{5} \\
\text { LTB }_{5} \\
\text { LTC }_{5}\end{array}$ \\
\hline
\end{tabular}

Tableau I - Métabolites actifs synthétisés à partir des AGPI. 